\section{Opening-Up Urged}

The International Committee for Future Accelerators (ICFA) representing high-energy labs on a regional basis stated after a special meeting in Vancouver that appropriate mechanisms be found to bring non-members into CERN's LHC project, and that "LHC be available for research by the world particle physics community". Conscious of a potential asymmetry, ICFA refered to linear colliders aimed at the established complimentary approach using electrons and positrons [ EN 24 (1993) 83] and noted that "in the not-to-distant future, accelerator specialists will complete the R\&D necessary to begin the design of an electron-positron collider capable of exploring the comparable mass region". This is done via an inter-regional collaboration with a memorandum of understanding where participants pledge "to admit all institutions that are prepared to make significant contributions to the R\&D effort. The participants further share a common vision of a facility that will be built as a worldwide collaboration".

The European Committee for Future Accelerators (ECFA) issued a statement at December's CERN Council also urging extended participation. An extraordinary Council meeting may be held this spring at about the time a sub-panel of the US Department of Energy's particle physics committee reports. Observers indicate that CERN hopes the vote on LHC will open at the June Council, and that ways to fund the project from CERN's annual budget are being sought (extra contributions would be supplementary).

The OECD (Organization for Economic Cooperation and Development) Megascience Forum [EN 23 (1993) 48] has set up a highenergy physics panel to examine the discipline's future, with an emphasis on international collaboration. Its report in April will focus on future facilities, notably several proposed medium-scale machines.

Fibres \& Components for System Applications: Present and Future University of Nice, 18-19 April 1994

Organized by COST Project 241 (Characterization of advanced fibres for the new photonic network) with Projects COST 240 (Techniques for modelling \& measuring advanced photonic communications components) \& COST 239 (Ultra high-capacity optical transmission networks).

Contact: C. Montes, Lab. de Physique de la Matière Condensée, Univ. de Nice-Sophia Antipolis, Parc Valrose, F-06108 Nice Cedex 2 [costws94@ naxos.unice.fr].

Tel.: +33935299 78; Fax: +3393529808

International Workshop
BIRTH OF THE UNIVERSE \&
FUNDAMENTAL PHYSICS
Rome, 18-21 May 1994

To review our understanding of the early Universe and large-scale structure and to examine whether the new astronomical observations need a new physics.

Registration fee (incl. proc.): \$US 150.(200.- after 28 Feb. 1994). EPS members: $20 \%$ discount.

For information: Ms. A. Restante, Observatorio Astronomico, Viale Parco Mellini, 84, I-00136 Rome (cosmo@vasrm4.astro.it) Tel.: +39-6-34 70 56; fax: +39-6-34 7802 .

\section{THE FACULTES UNIVERSITAIRES NOTRE-DAME DE LA PAIX}

\section{Namur, Belgium}

invites applications for a full-time position of

\section{CHARGE DE COURS}

at the Faculty of Sciences. The successful candidate will take over, in 1996, the Directorship of the Laboratoire de Spectroscopie Moléculaire de Surface (LASMOS), a member of the Belgian University Attraction Pole in Sciences of Interfacial and Mesoscopic Structures.

\section{Field of Research}

LASMOS is specialized in the use of electromagnetic probes (absorption-reflection spectroscopy, laser induced thermal desorption, resonant infra-red laser desorption, photovoltage spectroscopy and photoemission yield spectroscopy) and of nanoscopic probes (scanning tunneling and atomic force microscopies). Systems being investigated presently are metals and semiconductors, interfaces, fullerenes, zeolites, and biomolecules.

The Pole is conducting theoretical and experimental research on solid interfaces, aggregates and porous materials. Major equipment include extended computing facilities, a Van de Graaf accelerator, several XPS and HREELS machines, X-ray diffraction, solid-state NMR, MBE fabrication, and material synthesis and characterization.

\section{Qualifications}

Applicants must hold a PhD in experimental physics and have a substantial experience in the relevant field. They should show strong capabilities for conducting a team, initiating new research projects in collaboration with other laboratories and winning the support of external funding agencies. They must also be able to lecture efflciently in French, to first-year undergraduate students included.

\section{Information}

The appointment is tenured and for an indefinite period. Salary is fixed by Belgian federal law. More information can be obtained from the Chairman of the Physics Department, Prof. J.M. Gilles, FUNDP, rue de Bruxelles, 61, B-5000 Namur, Belgium (tel.: +32-81-72 47 14; fax: +32-81-72 47 07).

\section{Application}

Letters of application, including a curriculum vitae and a list of publications, should be sent before 1 May 1994 to R.P.M. Gilbert, Rector, FUNDP, rue de Bruxelles, 61, B-5000 Namur, Belgium.

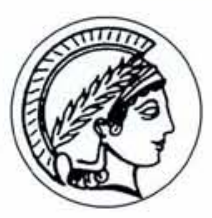

\section{Max-Planck-Institut für \\ Physik komplexer Systeme \\ in Dresden}

\section{Research Unit to be headed by a Junior Scientist}

The Max Planck Institute for Physics of Complex Systems seeks an outstanding candidate to lead a research group devoted to theoretical studies in the field of complex systems. The latter include - systems with nonlinear dynamics

$$
\text { - mesoscopic systems }
$$$$
\text { - many-body theory. }
$$

The successful candidate should have several years of post-doctoral experience and an outstanding record of achievement in one of these research areas.

The available position represents a five year appointment at a level equivalent to associate professor (C3). Additional salaries for one Ph.D. level scientist are guaranteed as well as appropriate space and funds for operation. Fellowships for Ph.D. students and guests are subject to negotiation.

Candidates should submit a curriculum vitae, a list of publications, a short description of their future research plan, and the names of three scientists who are familiar with the candidate's research accomplishments and scientific potential to:

$$
\text { Max-Planck-Gesellschaft - Generalverwaltung / Ref. III b }
$$

Postfach 101062 - 80084 München

Application deadline: 15 March 1994 